\title{
DUMMY VARIABLES AND PREDICTIVE TESTS FOR STRUCTURAL CHANGE *
}

\author{
Jean-Marie DUFOUR
}

University of Montréal, Montréal, P.Q. H3C $3 \mathrm{~J} 7$ Canada

Received 19 January 1981

We propose a dummy variable interpretation and an alternative proof of the predictive Chow test; we further show that it provides useful additional information on structural changes. We apply this approach to the St. Louis equation.

\section{Introduction}

An important way of assessing the reliability of an econometric model consists in checking whether it is stable over time [see Lucas (1976)]. Frequently this problem can be formalized as one of testing whether the coefficient vectors in two regressions (corresponding to disjoint subperiods) are equal. Namely, one considers

$\boldsymbol{y}_{i}=X_{i} \boldsymbol{\beta}_{i}+\boldsymbol{u}_{i}, \quad i=1,2$,

where $y_{i}$ is a $T_{i} \times 1$ vector of observations on a dependent variable, $X_{i}$ is a $T_{i} \times k$ non-stochastic matrix of explanatory variables, $\boldsymbol{\beta}_{i}$ is a vector of coefficients and $\boldsymbol{u}_{i}$ a $T_{i} \times 1$ vector of disturbances $(i=1,2)$. We assumc also that $\left(\boldsymbol{u}_{1}^{\prime}, \boldsymbol{u}_{2}^{\prime}\right)^{\prime} \sim N\left[\mathbf{0}, \sigma^{2} I_{T}\right]$, where $T=T_{1}+T_{2}$. The hypothesis to be tested is $H_{0}: \boldsymbol{\beta}_{1}=\boldsymbol{\beta}_{2}$. It is customary to assume further that $\operatorname{rank}\left(X_{1}\right)=k$ and to consider two distinct cases according to whether rank $\left(X_{2}\right)=k<$ $T_{2}$ or rand $\left(X_{2}\right)=T_{2} \leqslant k$. Tests known to econometricians as the "Chow tests' may then be applied. In the first case, a standard analysis-of-

* This research was supported by Grant 410-80-0501 of the S.S.H.R.C. of Canada and Quebec F.C.A.C. Grant EQ-1587. I am indebted to Marcel G. Dagenais and Marc J.I. Gaudry for several helpful comments.

0165-1765/81/0000-0000/\$02.50 (C) North-Holland 
covariance test may be used [see Chow (1960, p. 598)]. For the second case, a predictive test, was suggested by Chow (1960) and shown by him to be equivalent to a test based on the statistic:

$$
F_{1}=\frac{\left(S S_{0}-S S_{1}\right) / T_{2}}{S S_{1} /\left(T_{1}-k\right)}
$$

where $S S_{1}$ is the residual sum of squares based on the first $T_{1}$ observations and $S S_{0}$ is the residual sum of squares based on all $T$ observations; under the null hypothesis, this statistic follows an $F$ distribution with $\left(T_{2}, T_{1}-k\right)$ degrees of freedom. The latter test remains applicable when rank $\left(X_{2}\right)=k<T_{2}$ and may, even in this case, have a better power against $H_{0}$ than the analysis-of-covariance test [see Wilson (1978)].

On the other hand, it has been stressed by Gujarati $(1970 \mathrm{a}, \mathrm{b})$ that the analysis-of-covariance test can also be performed via the use of dummy variables and that the extra coefficients can provide useful additional information: although both methods yield an identical conclusion concerning the null hypothesis $H_{0}$, the latter has the advantage of automatically producing indications on the sources of difference between the two regressions, i.e., on which coefficients may differ. However, when the second sample is undersized $\left(T_{2}<k\right)$, the relevant explanatory-variable matrix does not have full column rand and thus Gujarati's procedure is not applicable. To the extent of our knowledge, no comparable interpretation has been given to the predictive test.

The first purpose of this note is to propose an interpretation of the predictive Chow test as a test on a set of dummy variables and to show that these can also provide revealing additional information. To be more specific, the predictive Chow test indicates whether there is, among the $T_{2}$ observations predicted, at least one observation whose mean is inconsistent with the model of the first $T_{1}$ observations; nevertheless, when $T_{2} \geqslant 2$, it does not point out which ones among the $T_{2}$ 'extra observations' deviate most strongly from this model and thus, when $H_{0}$ is rejected, may be causing the rejection of $H_{0}$. Of course, this knowledge can be of great use in assessing the importance and determining the causes of a structural change. We show below that a dummy variable approach provides a computationally very convenient method for performing predictive tests on each individual extra observation. The second purpose of this paper is to give a new and especially simple proof of the distribution of the predictive test statistic. Indeed, while the null distribution of the analysis-of-covariance test statistic can be obtained by show- 
ing it is a case of a linear hypothesis test on the coefficients a fullrank linear regression model, a similar proof is not apparently known for the predictive test and, consequently, a number of special proofs had to be devised for it [see Chow (1960), Fisher (1970) and Harvey (1976)]: we show below that the dummy variable interpretation of the same test provides a simple and natural way of making such a proof, similar to the one available for the first 'Chow test'. '

The alternative proof and interpretation of the predictive Chow test is described in section 2 . Its use in performing predictive tests on individual observations is discussed in section 3 . Results of an application to the St.Louis equation are reported in section 4.

\section{Alternative proof}

Let us define $y=\left(y_{1}^{\prime}, y_{2}^{\prime}\right)^{\prime}, \boldsymbol{u}=\left(\boldsymbol{u}_{1}^{\prime}, \boldsymbol{u}_{2}^{\prime}\right)^{\prime}, X=\left[X_{1}^{\prime}, X_{2}^{\prime}\right]^{\prime}$ and

$X^{*}=\left[\begin{array}{cc}X_{1} & 0 \\ X_{2} & I_{T_{2}}\end{array}\right]$,

where $I_{T_{2}}$ is the identity matrix of order $T_{2}$. We assume rank $\left(X_{1}\right)=k$ and rank $\left(X_{2}\right)=\min \left\{k, T_{2}\right\}$. It follows, since rank $\left(X_{1}\right)=k$ and rank $\left(I_{T_{2}}\right)=T_{2}$, that the $T \times\left(k+T_{2}\right)$ matrix $X$ has full column rank. Then let us consider the regression

$\boldsymbol{y}=X^{*}\left[\begin{array}{l}\boldsymbol{\beta} \\ \boldsymbol{\gamma}\end{array}\right]+\boldsymbol{u}$,

where $\boldsymbol{\beta}$ and $\gamma$ are vectors of coefficients of dimensions $k \times 1$ and $T_{2} \times 1$ respectively. The null hypothesis $H_{0}: \boldsymbol{\beta}_{1}=\boldsymbol{\beta}_{2} \equiv \boldsymbol{\beta}$ is equivalent to $\boldsymbol{\gamma}=\mathbf{0}$, and we can thus test it by testing $\gamma-0$ in (4). In other words, we add a dummy variable for each observation in the second regression and test whether all the coefficients of these dummy variables are zero. The standard $F$-test against $\boldsymbol{\gamma}=\mathbf{0}$ is based on the statistic

$F^{\prime}=\frac{\left(S S_{0}-S S_{1}^{\prime}\right) / T_{2}}{S S_{1}^{\prime} /\left(T-T_{2}-k\right)}$

1 Econometrics textbooks typically study inference only for the full-rank linear model: see, for example, Johnston (1972, ch. 5), Maddala (1977, ch. 8) or Theil (1971, ch. 3). Thus the proof obtained here may be especially convenient in teaching situations. 
where

$S S_{0}=\min _{\boldsymbol{\beta}}\|\boldsymbol{y}-X \boldsymbol{\beta}\|^{2}$,

$S S_{1}^{\prime}=\min _{\beta, \gamma}\left[\left\|y_{1}-X_{1} \beta\right\|^{2}+\left\|y_{2}-X_{2} \beta-\gamma\right\|^{2}\right]$.

Under the null hypothesis, $F^{\prime}$ follows an $F$ distribution with $\left(T_{2}, T-T_{2}\right.$ $-k$ ) degrees of freedom. Now, since we can always set $\hat{\boldsymbol{\gamma}}=\boldsymbol{y}_{2}-X_{2} \hat{\boldsymbol{\beta}}_{1}$, where $\hat{\boldsymbol{\beta}}_{1}$ is the value of $\boldsymbol{\beta}$ obtained while finding $S S_{1}^{\prime}$, we must have

$S S_{1}^{\prime}=\min _{\beta}\left\|y_{1}-X_{1} \beta\right\|^{2}=S S_{1}$,

hence, since $T-T_{2}-k=T_{1}-k$, we see that $F_{1}=F^{\prime}$ and thus $F_{1}$ follows an $F$-distribution with $\left(T_{2}, T_{1}-k\right)$ degrees of freedom.

Note that this proof of the distribution of $F_{1}$ is valid whether $T_{2} \leqslant k$ or $T_{2}>k$, and that it would also hold if, instead of $I_{T_{2}}$ in $X^{*}$, we had used any $T_{2} \times T_{2}$ non-singular matrix $Z$; in that case, one simply sets $\hat{\gamma}=Z^{-1}\left(y_{2}-X_{2} \hat{\beta}_{1}\right)$.

\section{Predictive tests on individual observations}

Let us now examine more closely what the coefficient vector $\gamma$ represents. If we rewrite eq. (4) in the form

$y_{t}=\boldsymbol{x}_{t}^{\prime} \boldsymbol{\beta}+\sum_{s=T_{1}+1}^{T} \gamma_{s} D_{t s}+u_{t}, \quad t=1, \ldots, T$,

where $\boldsymbol{x}_{t}^{\prime}=\left(x_{t 1}, \ldots, x_{t k}\right)$ is the $t$ th line of the matrix $X, \gamma=\left(\gamma_{T_{1+1}}, \ldots, \gamma_{T}\right)^{\prime}$ and

$$
\begin{aligned}
D_{t s} & =1, \quad t=s, \\
& =0, \quad t \neq s,
\end{aligned}
$$

we can see easily that

$\gamma_{s}=E\left(y_{s}\right)-\boldsymbol{x}_{s}^{\prime} \boldsymbol{\beta}, \quad s=T_{1}+1, \ldots, T$, 
i.e., $\gamma_{s}$ is the deviation of the actual mean of $y_{s}$ form the mean predicted by the 'common coefficient vector $\boldsymbol{\beta}$ '. These deviations can be estimated, in the process of performing the predictive Chow test, by estimating eq. (4) instead of the usual equation $\mathbf{y}=X \boldsymbol{\beta}+\boldsymbol{u}$. From the above proof, we can see that $\hat{\gamma}=y_{2}-X_{2} \hat{\boldsymbol{\beta}}_{1}$, where $\hat{\boldsymbol{\beta}}_{1}=\left(X_{1}^{\prime} X_{1}\right)^{-1} X_{1}^{\prime} \boldsymbol{y}_{1}$, and thus the covariance matrix of $\hat{\gamma}$ is $\sigma^{2} V$, where

$V=I_{T_{2}}+X_{2}\left(X_{1}^{\prime} X_{1}\right)^{-1} X_{2}^{\prime}$.

If eq. (4) is estimated using any standard regression package the estimate of $\sigma^{2} V$ produced will be of the form $s_{1}^{2} V$, where $s_{1}^{2}=S S_{1} /\left(T_{1}-k\right)$. Since $s_{1}^{2}$ is an unbiased estimate of $\sigma^{2}$, it is necessary that $V_{1}=V$. Furthermore, standard errors and $t$-statistics are usually produced automatically for each coefficient $\gamma_{s}$; from (10), the empirical standard error of $\hat{\gamma}_{s}$ is $s_{1} d_{s}$, where $d_{s}=\left[1+\boldsymbol{x}_{s}^{\prime}\left(X_{1}^{\prime} X_{1}\right)^{-1} \boldsymbol{x}_{s}\right]^{\frac{1}{2}}$, while the $t$-statistic associated with it is

$t_{s}=\left(y_{s}-\boldsymbol{x}_{s}^{\prime} \hat{\boldsymbol{\beta}}_{1}\right) / s_{1} d_{s}, \quad s=T_{1}+1, \ldots, T$,

under the null hypothesis $\gamma_{s}=0, t_{s}$ follows a Student- $t$ distribution with $T_{1}-k$ degrees of freedom. These statistics are the predictive test statistics for each observation $s=T_{1}+1, \ldots, T$, based on the parameter estimates obtained from the first $T_{1}$ observations. Their interest as diagnostic checks consists in pointing out which ones of these observations deviate most strongly from the model of the first $T_{1}$ observations.

\section{Application}

We applied the above technique to the 'St.Louis equation' in rate of change form, estimated over the period 1953/I-1976/IV [Carlson (1978, table IV)]; this equation relates changes in GNP to changes in the money stock and changes in high-employment expenditures in the U.S. Since the Almon polynomial lag restrictions used by Carlson were rejected by a preliminary test, no such constraints were imposed on the coefficients; in any case, the restricted and unrestricted models yield essentially the same policy implications. Using as subperiods 1953/I-1969/IV and 1970/I$1976 /$ IV, both Chow tests were found significant at levels lower than the conventional 5\% level. Furthermore, adding dummy variables for each observation in 1970/I-1976/IV, as suggested above, resulted in only 2 (among 28) of the prediction errors (1973/IV and 1975/III) with $t$-values 
significant at levels sensibly lower than 5\%. Indeed, if these 2 observations are dropped from the regression, both Chow statistics cease to be significant. Further details are given in a working paper [Dufour (1980)].

\section{Concluding remarks}

Regression (4) thus provides a computationally very convenient method for obtaining direct evidence on one of the main consequences of structural instability (large prediction errors) jointly with a whole array of predictive test statistics. Without the dummy variable method, one would need to perform $T_{2}$ extra regressions or to compute the $t_{s}$ statistics explicitly, which may be quite burdensome. Further, when $T_{2}>k$, the dummy variables considered above do not become identical with those used by Gujarati $(1970 \mathrm{a}, \mathrm{b})$ and give a different type of information, relating to the timing of structural change rather than coefficients. Thus the analysis-of-covariance test and the predictive test give complementary information and it may be useful to perform both tests (with dummies) whenever possible. Finally it can be pointed out that the simple and natural parametric interpretation given above of the predictive Chow test (as a test on the parameter vector $\gamma$ ) shows clearly how the predictive test is a test designed against a much wider set of alternatives than the analysis-of-covariance test (for $\boldsymbol{\beta}_{1} \neq \boldsymbol{\beta}_{2}$ is a special case of $\boldsymbol{\gamma} \neq 0$, while the converse does not hold); furthermore, this set-up makes straightforward the construction of Bayesian posterior odds in the case $T_{2}<k$, since all that is needed is putting a prior distribution on $\gamma .{ }^{2}$

\section{References}

Carlson, K.M., 1978, Does the St.Louis equation now believe in fiscal policy?, Federal Reserve Bank of St.Louis Review 60, no. 2, 13-19.

Chow, G.C., 1960, Tests of equality between sets of coefficients in two linear regressions, Econometrica 28, 591-603.

Dufour, J.-M., 1980, Predictive tests for structural change and the St.Louis equation, Cahier 8054 (University of Montréal, Montréal).

Fisher, F.M., 1970, Tests of equality between sets of coefficients in two linear regressions: An expository note, Econometrica 38, 361-366.

2 For the case rank $\left(X_{j}\right)=k<T_{i}, i=1,2$, such Bayesian posterior odds werc given by Zellner and Siow (1979). 
Gujarati, D., 1970a, Use of dummy variables in testing for equality between sets of coefficients in two linear regressions: A note, The American Statistician 24, no. I, 50-52.

Gujarati, D., 1970b, Use of dummy variables for equality between sets of coefficients in lincar regressions: A generalization, The American Statistician 24, no. 5, 18-22.

Harvey, A., 1976, An alternative proof and generalization of a test for structural change, The American Statistician 30, 122-123.

Johnston, J., 1972, Econometric methods, 2nd ed. (McGraw-Hill, New York).

Lucas, R.E. Jr., 1976, Econometric policy evaluation: A critique, in K. Brunner and A.H. Meltzer, eds., The Phillips curve and labor markets, Carnegie-Rochester conferences on public policy I (North-Holland, Amsterdam).

Maddala, G.S., 1977, Econometrics (McGraw-Hill, New York).

Theil, H., 1971, Principles of econometrics (Wiley, New York).

Wilson, A.L., 1978, When is the Chow test UMP?, The American Statistician 32, 66-68.

Zellner, A. and A. Siow, 1979, Bayesian posterior odds ratios for several frequently encountered hypothesis in regression analysis, Discussion Paper (Graduate School of Business, University of Chicago, IL). 